CRYSTALLOGRAPHIC COMMUNICATIONS

ISSN 2056-9890

Received 10 April 2018

Accepted 23 April 2018

Edited by L. Fabian, University of East Anglia, England

Keywords: crystal structure; azotropolone; azo group.

CCDC reference: 1838908

Supporting information: this article has supporting information at journals.iucr.org/e

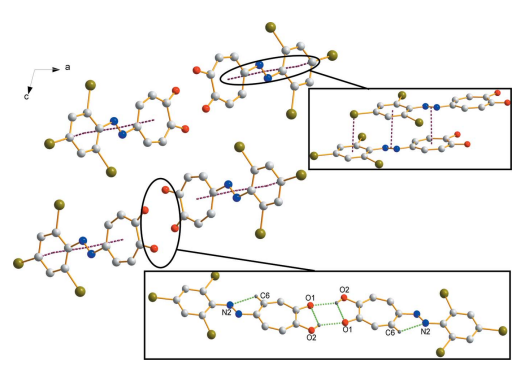

OPEN $\odot$ ACCESS

\section{Crystal structure of 5-[2-(2,4,6-tribromophenyl)- diazenyl]tropolone}

\author{
Tania N. Hill,* Kelsey L. Savig and Andreas Lemmerer
}

Molecular Sciences Institute, School of Chemistry, University of the Witwatersrand, PO WITS 2050, Johannesburg, South Africa. *Correspondence e-mail: tania.hill@gmail.com

The title compound \{systematic name: 2-hydroxy-5-[2-(2,4,6-tribromophenyl)diazen-1-yl]cyclohepta-2,4,6-trien-1-one $, \quad \mathrm{C}_{13} \mathrm{H}_{7} \mathrm{Br}_{3} \mathrm{~N}_{2} \mathrm{O}_{2}, \quad$ is essentially planar, with an r.m.s. deviation of $0.054 \AA$. The molecular structure is fixed in the azo tautomer by intramolecular $\mathrm{C}-\mathrm{H} \cdots \mathrm{N}$ interactions, with $\mathrm{O}-\mathrm{H} \cdots \mathrm{O}$ hydrogen bonds creating linked dimers. Charge-transfer interactions are observed, with the segregated stacks linked by $\mathrm{Br} \cdots \mathrm{Br}$ interactions.

\section{Chemical context}

In modern times, dyes have become an enormous market (approx. \$13.4 billion) with over one million tons of various dyes and pigments being produced each year, and their uses ranging from textiles, cosmetics, food coloring, printing inks to optical recording media. Azo dyes form one of the largest groups of synthetic chemical dyes with the chemical structure of these compounds featuring substituted aromatic rings that are joined by one or more azo groups $(R-\mathrm{N}=\mathrm{N}-R)$. The first of these dyes to be produced was aniline yellow, which contains an azo-substituted benzene group. Tropolone exhibits similar aromatic properties to benzene; as such, a study was undertaken to synthesize a series of azo-functionalized tropolones. A search of the Cambridge Structural Database (CSD; Groom et al., 2016) yielded twenty troponoid compounds with a mono-substituted 5-position. Of these, only eleven had the tropolone backbone, only two of which had an azo linking group. As part of this study, we report the crystal structure of the title compound, I (Fig. 1).<smiles>O=c1ccc(/N=N/c2c(Br)cc(Br)cc2Br)ccc1O</smiles>

\section{Structural commentary}

The title compound, I, shows no signs of azo-hydrazone tautomerization, a phenomenon known to the phenylazoderivatives, because of the stabilizing intramolecular interaction of the hydrogen (H6) atom of the tropolone ring with the nitrogen (N2) atom of the azo group (Table 1, Fig. 2). Similar to 5-phenylazotropolone (Hill et al., 2012), I is essen- 
Table 1

Hydrogen-bond geometry $\left(\AA,^{\circ}\right)$.

\begin{tabular}{lllll}
\hline$D-\mathrm{H} \cdots A$ & $D-\mathrm{H}$ & $\mathrm{H} \cdots A$ & $D \cdots A$ & $D-\mathrm{H} \cdots A$ \\
\hline $\mathrm{C} 6-\mathrm{H} 6 \cdots \mathrm{N} 2$ & 0.95 & 2.36 & $2.705(3)$ & 101 \\
$\mathrm{O} 2-\mathrm{H} 2 \cdots \mathrm{O} 1$ & 0.84 & 2.1 & $2.591(2)$ & 117 \\
$\mathrm{C} 3-\mathrm{H} 3 \cdots \mathrm{O} 2^{\mathrm{i}}$ & 0.95 & 2.54 & $3.241(3)$ & 131 \\
\hline
\end{tabular}

Symmetry code: (i) $-x+1, y-\frac{1}{2},-z+\frac{3}{2}$.

tially planar, with the dihedral angle between the least-squares planes $A(\mathrm{O} 1, \mathrm{O} 2, \mathrm{C} 1-\mathrm{C} 7, \mathrm{~N} 1)$ and $B(\mathrm{~N} 2, \mathrm{C} 11-\mathrm{C} 16)$ of $5.07(6)^{\circ}$ with an r.m.s. deviation of $0.054 \AA$. The largest variation from the molecular plane is for the phenyl carbon (C13) with a value of 0.096 (2) $\AA$. However, this planarity does not extend to the other azotropolones: 5-(4-ethoxyphenylazo)tropolone (Kubo et al., 2008) was found to be twisted with an angle of $27.6(1)^{\circ}$.

\section{Supramolecular features}

As with tropolone (Shimanouchi \& Sasada, 1973) along with the azotropolones, I forms centrosymmetric homodimers through an $\mathrm{O}-\mathrm{H} \cdots \mathrm{O}$ interaction (Table 1, Fig. 2). Steyl \& Roodt (2008) proposed a general range for $\mathrm{Br} \cdots \mathrm{Br}$ interactions, with distances between 3.6 and $4.6 \AA$ with an interaction angle of 40 to $180^{\circ}$. Two noteworthy $\mathrm{Br} \cdots \mathrm{Br}$ interactions are observed for compound I, firstly, a ring formed with the adjacent molecule with a distance of

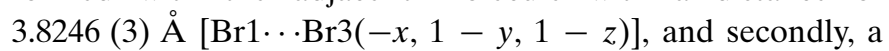
chain linking the molecules $\left[\mathrm{Br} 3 \cdots \mathrm{Br} 3\left(-x,-\frac{1}{2}+y, \frac{3}{2}-z\right)\right]$ with a distance of 3.6841 (4) $\AA$ (Fig. 3). Although the triangle interaction, which was observed by Steyl \& Roodt, was present for I, the distances are rather long [3.6441 (4), 3.8246 (3) and 4.0113 (4) $\AA$ ], but still fall into the range set.

The $\pi-\pi$ interactions that were observed for 5-phenylazotropolone and 5-(4-ethoxyphenylazo)tropolone are not observed for I. Instead, a $\pi \rightarrow \pi^{*}$ charge-transfer (CT) interaction between the aromatic phenyl and tropolone with the diazenyl group is seen (Fig. 2), with ring centroid to $\mathrm{N}=\mathrm{N}$ midpoint distances of 3.3463 (3) and 3.3415 (3) $\AA$, respectively. Additionally, a $\mathrm{Br} \cdots \pi$ interaction is found with a $\mathrm{Br}$ to benzene ring centroid distance of 3.4563 (3) $\AA$.

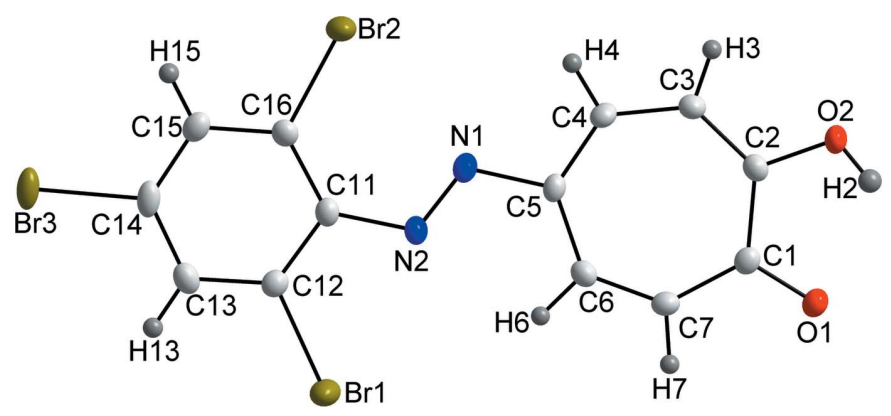

Figure 1

View of I with $50 \%$ probability displacement ellipsoids

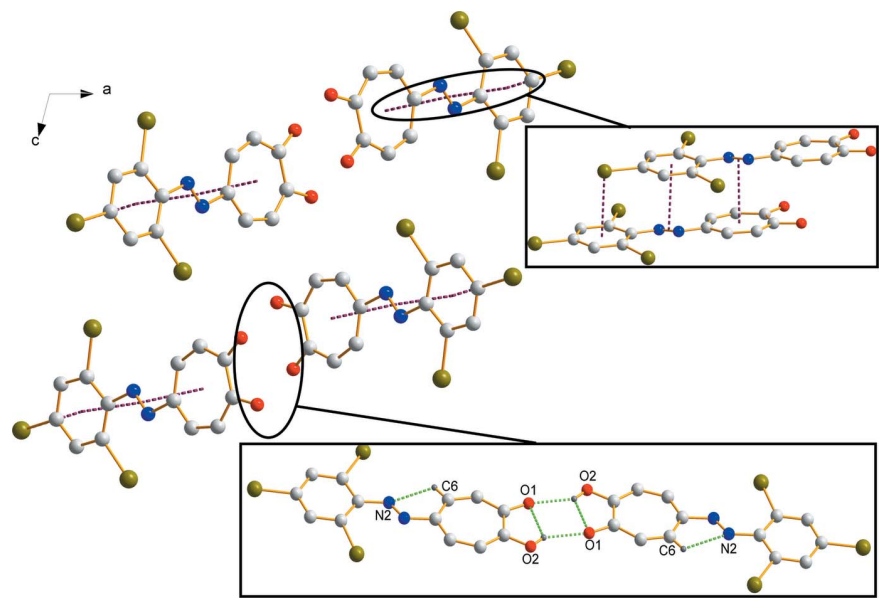

Figure 2

The packing of $\mathbf{I}$ as viewed along the $b$-axis. The top-right insert illustrates the $\mathrm{Br} \cdots \pi$ and $\pi \rightarrow \pi^{*} \mathrm{CT}$ interactions, while the bottom-right insert illustrates the $\mathrm{O}-\mathrm{H} \cdots \mathrm{O}$ and $\mathrm{C}-\mathrm{H} \cdots \mathrm{N}$ interactions as dashed bonds. Only selected hydrogen atoms are shown for clarity.

As a result of the observed $\mathrm{O}-\mathrm{H} \cdots \mathrm{O}, \mathrm{Br} \cdots \mathrm{Br}, \mathrm{Br} \cdots \pi$ and $\pi \rightarrow \pi^{*}$ CT interactions, the assembly of $\mathbf{I}$ is seen as forming segregated stacks along the $b$-axis direction (Fig. 2).

\section{Hirshfeld Surface Analysis}

Hirshfeld surface plots were generated for tropolone (CSD refcode: TROPOL10; Shimanouchi \& Sasada, 1973), 5-phenylazotropolone (CSD refcode: YDIVYZ; Hill et al., 2012) and I based on the crystallographic information file (CIF) using CrystalExplorer17.5 (Turner et al., 2017), to explore and compare the location of atom-to-atom short contacts along with the quantitative ratios of these interactions. Unfortunatly, the Hirshfeld surfaces for 5-(4-ethoxyphenylazo)tropolone could not be generated as the coordinates were not inputted into the CSD. The curvedness

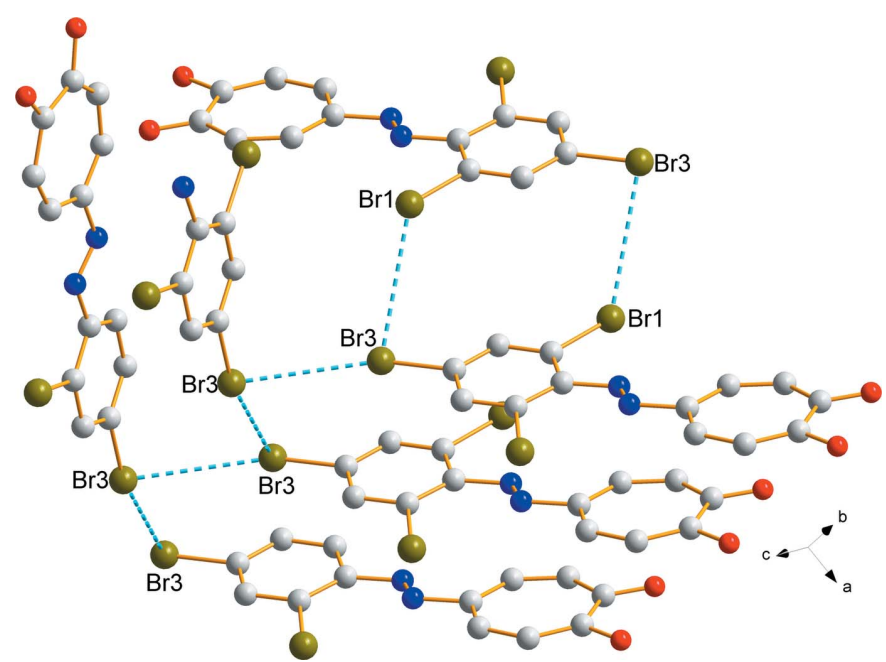

Figure 3

The $\mathrm{Br} \cdots \mathrm{Br}$ interactions of $\mathbf{I}$ illustrated with dashed bonds. Hydrogen atoms omitted for clarity. 


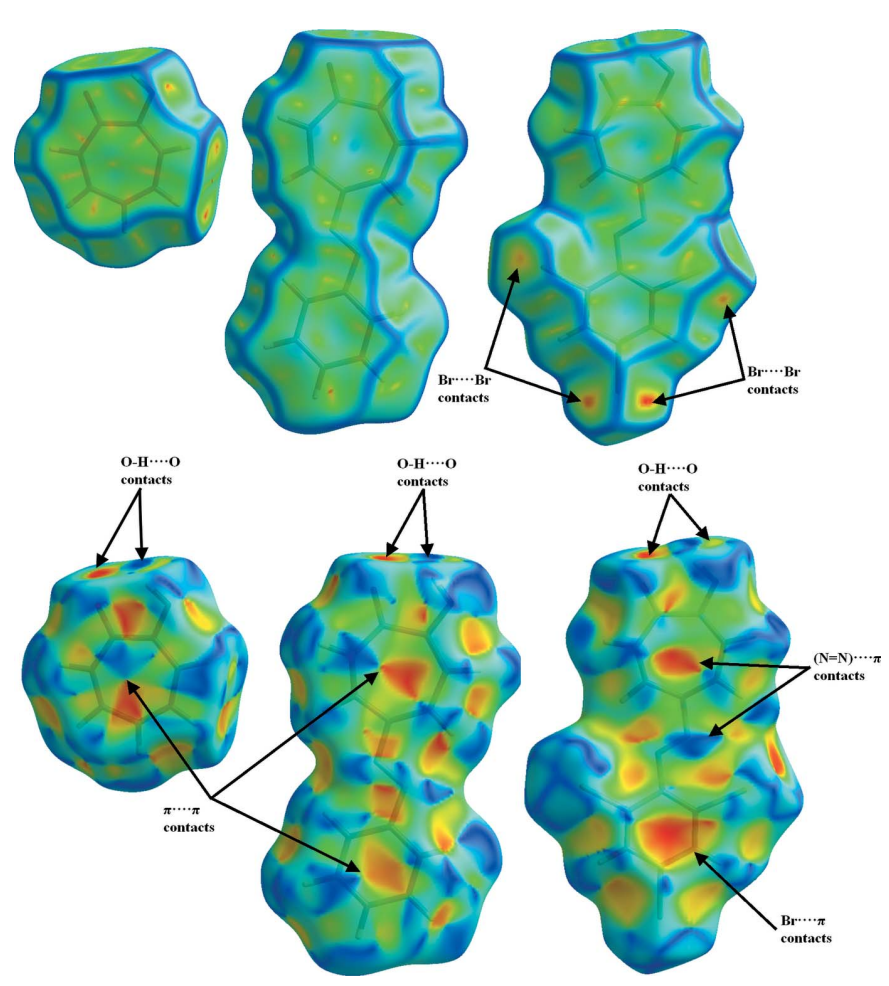

Figure 4

Hirshfeld surfaces for tropolone, 5-phenyltropolone and I, mapped with curvedness (top) and shape index (bottom).

plots of tropolone and 5-phenyltropolone show large regions of green. This is attributed to a relatively flat surface area (planar), whilst the blue regions illustrate areas of curvature (Fig. 4). For tropolone and 5-phenyltropolone, it can be seen that the molecules are essentially planar, as mentioned previously, with 5-phenyltropolone having a dihedral angle (between the least-squares planes of the tropolone and phenyl moieties) of $1.57(8)^{\circ}$. This is smaller than the corresponding angle found for $\mathbf{I}\left[5.07(6)^{\circ}\right]$, where the additional twist of the dihedral planes can clearly be seen in the curvedness plot, as there are additional blue regions which snake over the 'planar' surface of the molecule. The curvedness plots of the compounds show flat surface areas, which is consistent with the planar packing arrangement that has been observed for both tropolone and 5-phenylazotropolone. It is interesting to note that in the curvedness plots, the $\mathrm{Br} \cdots \mathrm{Br}$ interactions are clearly visible as curved (red) regions and contribute $9 \%$ to the total surface area. These interactions are mirrored in the shape-index plots but are a little harder to observe.

On the shape-index surface plots for tropolone, 5-phenylazotropolone and I (Fig. 4), convex blue regions represent donor groups, whilst the red concave regions are the acceptor groups. The $\pi-\pi$ interactions are generally indicated by adjacent red and blue triangles. These triangles are clearly observed for both tropolone (17.5\% surface contribution) and 5-phenyltropolone (10.5\% surface contribution), whereas this triangle formation was not found for $\mathbf{I}$, further supporting the finding of no $\pi-\pi$ interactions. As mentioned in the Supramolecular features section, I is seen to have both $\mathrm{Br} \cdots \pi(7.1 \%$ surface contribution) and CT (9.2\% surface contribution)
Table 2

Experimental details.

\begin{tabular}{|c|c|}
\hline \multicolumn{2}{|l|}{ Crystal data } \\
\hline Chemical formula & $\mathrm{C}_{13} \mathrm{H}_{7} \mathrm{Br}_{3} \mathrm{~N}_{2} \mathrm{O}_{2}$ \\
\hline$M_{\mathrm{r}}$ & 462.94 \\
\hline Crystal system, space group & Monoclinic, $P 2_{1} / c$ \\
\hline Temperature (K) & 173 \\
\hline$a, b, c(\AA)$ & $18.1742(3), 4.7885(1), 16.0308(3)$ \\
\hline$\beta\left(^{\circ}\right)$ & $104.560(1)$ \\
\hline$V\left(\AA^{3}\right)$ & $1350.31(4)$ \\
\hline$Z$ & 4 \\
\hline Radiation type & Мо $K \alpha$ \\
\hline$\mu\left(\mathrm{mm}^{-1}\right)$ & 8.96 \\
\hline Crystal size $(\mathrm{mm})$ & $0.4 \times 0.12 \times 0.07$ \\
\hline \multicolumn{2}{|l|}{ Data collection } \\
\hline Diffractometer & $\begin{array}{l}\text { Bruker APEXII CCD area } \\
\text { detector }\end{array}$ \\
\hline Absorption correction & $\begin{array}{l}\text { Multi-scan (SADABS; Bruker, } \\
\text { 2004) }\end{array}$ \\
\hline$T_{\min }, T_{\max }$ & $0.124,0.573$ \\
\hline $\begin{array}{l}\text { No. of measured, independent and } \\
\text { observed }[I>2 \sigma(I)] \text { reflections }\end{array}$ & $27599,3258,2744$ \\
\hline$R_{\text {int }}$ & 0.055 \\
\hline$(\sin \theta / \lambda)_{\max }\left(\AA^{-1}\right)$ & 0.661 \\
\hline \multicolumn{2}{|l|}{ Refinement } \\
\hline$R\left[F^{2}>2 \sigma\left(F^{2}\right)\right], w R\left(F^{2}\right), S$ & $0.023,0.051,1.02$ \\
\hline No. of reflections & 3258 \\
\hline No. of parameters & 182 \\
\hline $\mathrm{H}$-atom treatment & $\mathrm{H}$-atom parameters constrained \\
\hline$\Delta \rho_{\max }, \Delta \rho_{\min }\left(\mathrm{e} \AA^{-3}\right)$ & $0.77,-0.68$ \\
\hline
\end{tabular}

Computer programs: APEX2 (Bruker, 2005), SAINT-Plus and XPREP (Bruker, 2004), SHELXS97 and SHELXL97 (Sheldrick, 2008), DIAMOND (Brandenburg \& Putz, 2005) and WinGX (Farrugia, 2012).

interactions (Fig. 2). This is further supported by the Hirshfeld shape-index surface illustrating the blue region of the donor azo group and the red accepting region of the tropolone moiety (Fig. 4).

\section{Synthesis and crystallization}

The reagents were commercially obtained and used without further purification.

Sodium nitrite $(1.4 \mathrm{mmol})$ dissolved in water $\left(1 \mathrm{~cm}^{3}\right)$ was added dropwise to a solution containing 2,4,6-tribromoaniline (1.6 mmol), hydrochloric acid $\left(2 \mathrm{~cm}^{3}\right.$, conc.) and water $\left(7 \mathrm{~cm}^{3}\right)$. Upon cooling the resultant mixture to $c a 277 \mathrm{~K}$, it was added slowly to a solution of sodium hydroxide $(1.8 \mathrm{mmol})$ and tropolone $(1.6 \mathrm{mmol})$ in water $\left(4 \mathrm{~cm}^{3}\right)$, keeping the temperature below $278 \mathrm{~K}$. The resulting solution was stirred for $30 \mathrm{~min}$., filtered and air-dried. Crystals suitable for X-ray diffraction were obtained by recrystallization with $\mathrm{CHCl}_{3}$.

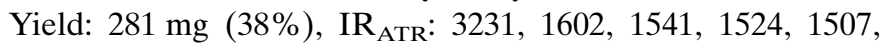
1439, 1411, 1439, 1304, 1258, 1190, 1052, 872, 852, 799, 730, 705, $669,620,604,546,490 \mathrm{~cm}^{-1}$.

\section{Refinement}

Crystal data, data collection and structure refinement details are summarized in Table 2. All hydrogen atoms were positioned geometrically and refined using a riding model, with $\mathrm{C}-\mathrm{H}=0.95 \AA, U_{\text {iso }}(\mathrm{H})=1.2 U_{\text {eq }}(\mathrm{C})$ for aromatic $\mathrm{H}$ atoms and 
with $\mathrm{O}-\mathrm{H}=0.84 \AA$, $U_{\text {iso }}(\mathrm{H})=1.5 U_{\text {eq }}(\mathrm{O})$ for the hydroxy $\mathrm{H}$ atom.

\section{Funding information}

The University of the Witwatersand and the Molecular Sciences Institute are thanked for providing the infrastructure and financial support required to do this work.

\section{References}

Brandenburg, K. \& Putz, H. (2005). DIAMOND. Crystal Impact GbR, Bonn, Germany.

Bruker (2004). SAINT-Plus, SADABS and XPREP. Bruker AXS Inc., Madison, WI, USA.
Bruker (2005). APEX2. Bruker AXS Inc., Madison, WI, USA.

Farrugia, L. J. (2012). J. Appl. Cryst. 45, 849-854.

Groom, C. R., Bruno, I. J., Lightfoot, M. P. \& Ward, S. C. (2016). Acta Cryst. B72, 171-179.

Hill, T. N., Mangwaela, M. S. \& Steyl, G. (2012). Acta Cryst. E68, o941.

Kubo, K., Yamamoto, E., Kakihara, Y., Matsumoto, T. \& Mori, A. (2008). J. Oleo Sci. 57, 513-519.

Sheldrick, G. M. (2008). Acta Cryst. A64, 112-122.

Shimanouchi, H. \& Sasada, Y. (1973). Acta Cryst. B29, 81-90.

Steyl, G. \& Roodt, A. (2008). Models, Mysteries and Magic of Molecules, J. C. A. Boeyens and J. F. Olgilvie, pp. 325-340. Netherlands: Springer.

Turner, M. J., McKinnon, J. J., Wolff, S. K., Grimwood, D. J., Spackman, P. R., Jayatilaka, D. \& Spackman, M. A. (2017). CrystalExplorer17. The University of Western Australia. 


\section{supporting information}

Acta Cryst. (2018). E74, 709-712 [https://doi.org/10.1107/S2056989018006151]

\section{Crystal structure of 5-[2-(2,4,6-tribromophenyl) diazenyl]tropolone}

\section{Tania N. Hill, Kelsey L. Savig and Andreas Lemmerer}

Computing details

Data collection: APEX2 (Bruker, 2005); cell refinement: SAINT-Plus (Bruker, 2004); data reduction: SAINT-Plus (Bruker, 2004) and XPREP (Bruker, 2004); program(s) used to solve structure: SHELXS97 (Sheldrick, 2008); program(s) used to refine structure: SHELXL97 (Sheldrick, 2008); molecular graphics: DIAMOND (Brandenburg \& Putz, 2005); software used to prepare material for publication: Win $G X$ (Farrugia, 2012).

2-Hydroxy-5-[2-(2,4,6-tribromophenyl)diazen-1-yl]cyclohepta-2,4,6-trien-1-one

Crystal data

$\mathrm{C}_{13} \mathrm{H}_{7} \mathrm{Br}_{3} \mathrm{~N}_{2} \mathrm{O}_{2}$

$M_{r}=462.94$

Monoclinic, $P 2_{1} / c$

Hall symbol: $-\mathrm{P} 2 \mathrm{ybc}$

$a=18.1742(3) \AA$

$b=4.7885(1) \AA$

$c=16.0308(3) \AA$

$\beta=104.560(1)^{\circ}$

$V=1350.31(4) \AA^{3}$

$Z=4$

\section{Data collection}

Bruker APEXII CCD area detector diffractometer

Radiation source: fine-focus sealed tube Graphite monochromator

Detector resolution: 512 pixels $\mathrm{mm}^{-1}$ $\varphi$ and $\omega$ scans

Absorption correction: multi-scan

(SADABS; Bruker, 2004)

$T_{\min }=0.124, T_{\max }=0.573$

Refinement

Refinement on $F^{2}$

Least-squares matrix: full

$R\left[F^{2}>2 \sigma\left(F^{2}\right)\right]=0.023$

$w R\left(F^{2}\right)=0.051$

$S=1.01$

3258 reflections

182 parameters
$F(000)=880$

$D_{\mathrm{x}}=2.277 \mathrm{Mg} \mathrm{m}^{-3}$

Mo $K \alpha$ radiation, $\lambda=0.71073 \AA$

Cell parameters from 8182 reflections

$\theta=2.3-28.2^{\circ}$

$\mu=8.96 \mathrm{~mm}^{-1}$

$T=173 \mathrm{~K}$

Plate, red

$0.4 \times 0.12 \times 0.07 \mathrm{~mm}$

27599 measured reflections

3258 independent reflections

2744 reflections with $I>2 \sigma(I)$

$R_{\text {int }}=0.055$

$\theta_{\max }=28^{\circ}, \theta_{\min }=2.3^{\circ}$

$h=-24 \rightarrow 24$

$k=-6 \rightarrow 6$

$l=-20 \rightarrow 21$

0 restraints

H-atom parameters constrained

$w=1 /\left[\sigma^{2}\left(F_{\mathrm{o}}^{2}\right)+(0.0151 P)^{2}+1.5233 P\right]$

where $P=\left(F_{\mathrm{o}}^{2}+2 F_{\mathrm{c}}{ }^{2}\right) / 3$

$(\Delta / \sigma)_{\max }=0.001$

$\Delta \rho_{\max }=0.77 \mathrm{e} \AA^{-3}$

$\Delta \rho_{\min }=-0.68$ e $\AA^{-3}$ 


\section{Special details}

Geometry. All esds (except the esd in the dihedral angle between two 1.s. planes) are estimated using the full covariance matrix. The cell esds are taken into account individually in the estimation of esds in distances, angles and torsion angles; correlations between esds in cell parameters are only used when they are defined by crystal symmetry. An approximate (isotropic) treatment of cell esds is used for estimating esds involving l.s. planes.

Fractional atomic coordinates and isotropic or equivalent isotropic displacement parameters $\left(\AA^{2}\right)$

\begin{tabular}{lllll}
\hline & $x$ & $y$ & $z$ & $U_{\text {iso }} / U_{\text {eq }}$ \\
\hline C1 & $0.41630(13)$ & $1.5974(5)$ & $0.51392(14)$ & $0.0173(5)$ \\
C2 & $0.45286(13)$ & $1.6351(5)$ & $0.60559(14)$ & $0.0171(4)$ \\
C3 & $0.43788(13)$ & $1.5012(5)$ & $0.67536(15)$ & $0.0189(5)$ \\
H3 & 0.4684 & 1.5592 & 0.7298 & $0.023^{*}$ \\
C4 & $0.38490(13)$ & $1.2934(5)$ & $0.67894(14)$ & $0.0191(5)$ \\
H4 & 0.3844 & 1.2338 & 0.7353 & $0.023^{*}$ \\
C5 & $0.33337(12)$ & $1.1622(5)$ & $0.61327(14)$ & $0.0163(4)$ \\
C6 & $0.32247(13)$ & $1.2070(5)$ & $0.52358(14)$ & $0.0181(5)$ \\
H6 & 0.285 & 1.0929 & 0.4873 & $0.022^{*}$ \\
C7 & $0.35821(13)$ & $1.3907(5)$ & $0.48232(15)$ & $0.0190(5)$ \\
H7 & 0.3419 & 1.3811 & 0.4213 & $0.023^{*}$ \\
C11 & $0.20015(13)$ & $0.6200(5)$ & $0.62034(14)$ & $0.0171(4)$ \\
C12 & $0.14250(13)$ & $0.4963(5)$ & $0.55595(14)$ & $0.0184(5)$ \\
C13 & $0.09308(13)$ & $0.2960(5)$ & $0.57313(15)$ & $0.0208(5)$ \\
H13 & 0.0542 & 0.2182 & 0.5282 & $0.025^{*}$ \\
C14 & $0.10233(13)$ & $0.2135(5)$ & $0.65781(16)$ & $0.0213(5)$ \\
C15 & $0.15835(14)$ & $0.3243(5)$ & $0.72361(15)$ & $0.0208(5)$ \\
H15 & 0.1639 & 0.263 & 0.7812 & $0.025^{*}$ \\
C16 & $0.20691(13)$ & $0.5268(5)$ & $0.70536(14)$ & $0.0186(5)$ \\
N1 & $0.28798(11)$ & $0.9647(4)$ & $0.64541(13)$ & $0.0221(4)$ \\
N2 & $0.24557(11)$ & $0.8171(4)$ & $0.58994(12)$ & $0.0200(4)$ \\
O1 & $0.43793(10)$ & $1.7538(4)$ & $0.46231(10)$ & $0.0230(4)$ \\
O2 & $0.50700(10)$ & $1.8299(3)$ & $0.62292(11)$ & $0.0225(4)$ \\
H2 & 0.511 & 1.9037 & 0.5767 & $0.034^{*}$ \\
Br1 & $0.128145(15)$ & $0.60750(6)$ & $0.439523(15)$ & $0.02966(7)$ \\
Br2 & $0.282839(16)$ & $0.65030(6)$ & $0.802286(15)$ & $0.03009(8)$ \\
Br3 & $0.036554(14)$ & $-0.06019(5)$ & $0.684700(18)$ & $0.02738(7)$ \\
& & & &
\end{tabular}

Atomic displacement parameters $\left(\AA^{2}\right)$

\begin{tabular}{lllllll}
\hline & $U^{11}$ & $U^{22}$ & $U^{33}$ & $U^{12}$ & $U^{13}$ & $U^{23}$ \\
\hline C1 & $0.0184(11)$ & $0.0153(11)$ & $0.0192(11)$ & $0.0027(9)$ & $0.0069(9)$ & $0.0010(9)$ \\
C2 & $0.0160(11)$ & $0.0147(10)$ & $0.0201(11)$ & $0.0005(8)$ & $0.0038(9)$ & $-0.0016(9)$ \\
C3 & $0.0193(11)$ & $0.0198(11)$ & $0.0164(11)$ & $-0.0005(9)$ & $0.0021(9)$ & $0.0002(9)$ \\
C4 & $0.0199(12)$ & $0.0211(12)$ & $0.0155(11)$ & $0.0000(9)$ & $0.0029(9)$ & $0.0042(9)$ \\
C5 & $0.0150(11)$ & $0.0146(10)$ & $0.0206(11)$ & $0.0007(9)$ & $0.0068(9)$ & $0.0025(9)$ \\
C6 & $0.0179(11)$ & $0.0156(11)$ & $0.0195(11)$ & $-0.0001(9)$ & $0.0022(9)$ & $-0.0011(9)$ \\
C7 & $0.0208(12)$ & $0.0202(12)$ & $0.0152(11)$ & $0.0008(9)$ & $0.0030(9)$ & $0.0006(9)$ \\
C11 & $0.0164(11)$ & $0.0142(11)$ & $0.0217(11)$ & $0.0004(8)$ & $0.0064(9)$ & $0.0004(9)$
\end{tabular}




\begin{tabular}{lllllll}
$\mathrm{C} 12$ & $0.0178(11)$ & $0.0190(11)$ & $0.0191(11)$ & $0.0018(9)$ & $0.0060(9)$ & $0.0013(9)$ \\
$\mathrm{C} 13$ & $0.0183(12)$ & $0.0177(11)$ & $0.0266(12)$ & $-0.0010(9)$ & $0.0060(10)$ & $-0.0033(9)$ \\
$\mathrm{C} 14$ & $0.0200(12)$ & $0.0135(11)$ & $0.0347(13)$ & $0.0007(9)$ & $0.0146(10)$ & $0.0009(10)$ \\
$\mathrm{C} 15$ & $0.0248(12)$ & $0.0179(11)$ & $0.0221(12)$ & $0.0019(10)$ & $0.0102(10)$ & $0.0021(9)$ \\
$\mathrm{C} 16$ & $0.0203(12)$ & $0.0165(11)$ & $0.0195(11)$ & $0.0000(9)$ & $0.0058(9)$ & $-0.0020(9)$ \\
$\mathrm{N} 1$ & $0.0217(10)$ & $0.0220(10)$ & $0.0224(10)$ & $-0.0043(8)$ & $0.0055(8)$ & $0.0043(8)$ \\
$\mathrm{N} 2$ & $0.0185(10)$ & $0.0199(10)$ & $0.0217(10)$ & $-0.0047(8)$ & $0.0051(8)$ & $0.0012(8)$ \\
$\mathrm{O} 1$ & $0.0287(9)$ & $0.0202(8)$ & $0.0207(8)$ & $-0.0039(7)$ & $0.0076(7)$ & $0.0027(7)$ \\
$\mathrm{O} 2$ & $0.0253(9)$ & $0.0214(8)$ & $0.0213(8)$ & $-0.0088(7)$ & $0.0070(7)$ & $0.0012(7)$ \\
$\mathrm{Br} 1$ & $0.02972(14)$ & $0.03815(15)$ & $0.01879(12)$ & $-0.00894(11)$ & $0.00180(10)$ & $0.00365(10)$ \\
$\mathrm{Br} 2$ & $0.04013(16)$ & $0.03091(14)$ & $0.01657(12)$ & $-0.01094(11)$ & $0.00216(10)$ & $0.00090(10)$ \\
$\mathrm{Br} 3$ & $0.02564(13)$ & $0.01662(12)$ & $0.04556(16)$ & $-0.00247(10)$ & $0.01955(11)$ & $0.00277(11)$ \\
\hline
\end{tabular}

Geometric parameters $\left(\AA,{ }^{\circ}\right)$

\begin{tabular}{|c|c|c|c|}
\hline $\mathrm{C} 1-\mathrm{O} 1$ & $1.250(3)$ & $\mathrm{C} 11-\mathrm{C} 12$ & $1.404(3)$ \\
\hline $\mathrm{C} 1-\mathrm{C} 7$ & $1.443(3)$ & $\mathrm{C} 11-\mathrm{C} 16$ & $1.410(3)$ \\
\hline $\mathrm{C} 1-\mathrm{C} 2$ & $1.464(3)$ & $\mathrm{C} 11-\mathrm{N} 2$ & $1.419(3)$ \\
\hline $\mathrm{C} 2-\mathrm{O} 2$ & $1.333(3)$ & $\mathrm{C} 12-\mathrm{C} 13$ & $1.388(3)$ \\
\hline $\mathrm{C} 2-\mathrm{C} 3$ & $1.375(3)$ & $\mathrm{C} 12-\mathrm{Br} 1$ & $1.895(2)$ \\
\hline $\mathrm{C} 3-\mathrm{C} 4$ & $1.396(3)$ & $\mathrm{C} 13-\mathrm{C} 14$ & $1.383(3)$ \\
\hline $\mathrm{C} 3-\mathrm{H} 3$ & 0.95 & $\mathrm{C} 13-\mathrm{H} 13$ & 0.95 \\
\hline $\mathrm{C} 4-\mathrm{C} 5$ & $1.373(3)$ & $\mathrm{C} 14-\mathrm{C} 15$ & $1.375(3)$ \\
\hline $\mathrm{C} 4-\mathrm{H} 4$ & 0.95 & $\mathrm{C} 14-\mathrm{Br} 3$ & $1.895(2)$ \\
\hline $\mathrm{C} 5-\mathrm{C} 6$ & $1.417(3)$ & $\mathrm{C} 15-\mathrm{C} 16$ & $1.391(3)$ \\
\hline $\mathrm{C} 5-\mathrm{N} 1$ & $1.433(3)$ & C15-H15 & 0.95 \\
\hline $\mathrm{C} 6-\mathrm{C} 7$ & $1.360(3)$ & $\mathrm{C} 16-\mathrm{Br} 2$ & $1.895(2)$ \\
\hline C6-H6 & 0.95 & $\mathrm{~N} 1-\mathrm{N} 2$ & $1.241(3)$ \\
\hline $\mathrm{C} 7-\mathrm{H} 7$ & 0.95 & $\mathrm{O} 2-\mathrm{H} 2$ & 0.84 \\
\hline $\mathrm{O} 1-\mathrm{C} 1-\mathrm{C} 7$ & $120.2(2)$ & $\mathrm{C} 12-\mathrm{C} 11-\mathrm{C} 16$ & $116.4(2)$ \\
\hline $\mathrm{O} 1-\mathrm{C} 1-\mathrm{C} 2$ & $116.8(2)$ & $\mathrm{C} 12-\mathrm{C} 11-\mathrm{N} 2$ & $114.79(19)$ \\
\hline $\mathrm{C} 7-\mathrm{C} 1-\mathrm{C} 2$ & $123.0(2)$ & $\mathrm{C} 16-\mathrm{C} 11-\mathrm{N} 2$ & $128.8(2)$ \\
\hline $\mathrm{O} 2-\mathrm{C} 2-\mathrm{C} 3$ & $116.3(2)$ & $\mathrm{C} 13-\mathrm{C} 12-\mathrm{C} 11$ & $123.1(2)$ \\
\hline $\mathrm{O} 2-\mathrm{C} 2-\mathrm{C} 1$ & $114.9(2)$ & $\mathrm{C} 13-\mathrm{C} 12-\mathrm{Br} 1$ & $117.00(17)$ \\
\hline $\mathrm{C} 3-\mathrm{C} 2-\mathrm{C} 1$ & $128.8(2)$ & $\mathrm{C} 11-\mathrm{C} 12-\mathrm{Br} 1$ & $119.88(17)$ \\
\hline $\mathrm{C} 2-\mathrm{C} 3-\mathrm{C} 4$ & $130.2(2)$ & $\mathrm{C} 14-\mathrm{C} 13-\mathrm{C} 12$ & $117.9(2)$ \\
\hline $\mathrm{C} 2-\mathrm{C} 3-\mathrm{H} 3$ & 114.9 & $\mathrm{C} 14-\mathrm{C} 13-\mathrm{H} 13$ & 121.1 \\
\hline $\mathrm{C} 4-\mathrm{C} 3-\mathrm{H} 3$ & 114.9 & $\mathrm{C} 12-\mathrm{C} 13-\mathrm{H} 13$ & 121.1 \\
\hline $\mathrm{C} 5-\mathrm{C} 4-\mathrm{C} 3$ & $129.7(2)$ & $\mathrm{C} 15-\mathrm{C} 14-\mathrm{C} 13$ & $121.8(2)$ \\
\hline $\mathrm{C} 5-\mathrm{C} 4-\mathrm{H} 4$ & 115.1 & $\mathrm{C} 15-\mathrm{C} 14-\mathrm{Br} 3$ & $118.70(18)$ \\
\hline $\mathrm{C} 3-\mathrm{C} 4-\mathrm{H} 4$ & 115.1 & $\mathrm{C} 13-\mathrm{C} 14-\mathrm{Br} 3$ & $119.53(18)$ \\
\hline $\mathrm{C} 4-\mathrm{C} 5-\mathrm{C} 6$ & $127.1(2)$ & $\mathrm{C} 14-\mathrm{C} 15-\mathrm{C} 16$ & $119.6(2)$ \\
\hline $\mathrm{C} 4-\mathrm{C} 5-\mathrm{N} 1$ & $111.70(19)$ & $\mathrm{C} 14-\mathrm{C} 15-\mathrm{H} 15$ & 120.2 \\
\hline $\mathrm{C} 6-\mathrm{C} 5-\mathrm{N} 1$ & $121.2(2)$ & $\mathrm{C} 16-\mathrm{C} 15-\mathrm{H} 15$ & 120.2 \\
\hline $\mathrm{C} 7-\mathrm{C} 6-\mathrm{C} 5$ & $129.0(2)$ & $\mathrm{C} 15-\mathrm{C} 16-\mathrm{C} 11$ & $121.2(2)$ \\
\hline $\mathrm{C} 7-\mathrm{C} 6-\mathrm{H} 6$ & 115.5 & $\mathrm{C} 15-\mathrm{C} 16-\mathrm{Br} 2$ & $114.52(17)$ \\
\hline $\mathrm{C} 5-\mathrm{C} 6-\mathrm{H} 6$ & 115.5 & $\mathrm{C} 11-\mathrm{C} 16-\mathrm{Br} 2$ & $124.22(17)$ \\
\hline
\end{tabular}




$\begin{array}{llll}\mathrm{C} 6-\mathrm{C} 7-\mathrm{C} 1 & 132.0(2) & \mathrm{N} 2-\mathrm{N} 1-\mathrm{C} 5 & 115.36(19) \\ \mathrm{C} 6-\mathrm{C} 7-\mathrm{H} 7 & 114 & \mathrm{~N} 1-\mathrm{N} 2-\mathrm{C} 11 & 116.30(19) \\ \mathrm{C} 1-\mathrm{C} 7-\mathrm{H} 7 & 114 & \mathrm{C} 2-\mathrm{O} 2-\mathrm{H} 2 & 109.5 \\ \mathrm{O} 1-\mathrm{C} 1-\mathrm{C} 2-\mathrm{O} 2 & & \mathrm{C} 11-\mathrm{C} 12-\mathrm{C} 13-\mathrm{C} 14 & -0.9(4) \\ \mathrm{C} 7-\mathrm{C} 1-\mathrm{C} 2-\mathrm{O} 2 & -1.7(3) & \mathrm{B} 1-\mathrm{C} 12-\mathrm{C} 13-\mathrm{C} 14 & -179.57(17) \\ \mathrm{O} 1-\mathrm{C} 1-\mathrm{C} 2-\mathrm{C} 3 & 178.0(2) & \mathrm{C} 12-\mathrm{C} 13-\mathrm{C} 14-\mathrm{C} 15 & 0.0(4) \\ \mathrm{C} 7-\mathrm{C} 1-\mathrm{C} 2-\mathrm{C} 3 & 177.0(2) & \mathrm{C} 12-\mathrm{C} 13-\mathrm{C} 14-\mathrm{Br} 3 & -179.80(17) \\ \mathrm{O} 2-\mathrm{C} 2-\mathrm{C} 3-\mathrm{C} 4 & -3.4(4) & \mathrm{C} 13-\mathrm{C} 14-\mathrm{C} 15-\mathrm{C} 16 & 0.6(4) \\ \mathrm{C} 1-\mathrm{C} 2-\mathrm{C} 3-\mathrm{C} 4 & 179.1(2) & \mathrm{Br} 3-\mathrm{C} 14-\mathrm{C} 15-\mathrm{C} 16 & -179.58(17) \\ \mathrm{C} 2-\mathrm{C} 3-\mathrm{C} 4-\mathrm{C} 5 & 0.5(4) & \mathrm{C} 14-\mathrm{C} 15-\mathrm{C} 16-\mathrm{C} 11 & -17(4) \\ \mathrm{C} 3-\mathrm{C} 4-\mathrm{C} 5-\mathrm{C} 6 & 0.9(5) & \mathrm{C} 14-\mathrm{C} 15-\mathrm{C} 16-\mathrm{Br} 2 & -0.4(3) \\ \mathrm{C} 3-\mathrm{C} 4-\mathrm{C} 5-\mathrm{N} 1 & 0.9(4) & \mathrm{N} 2-\mathrm{C} 11-\mathrm{C} 16-\mathrm{C} 15 & -178.1(2) \\ \mathrm{C} 4-\mathrm{C} 5-\mathrm{C} 6-\mathrm{C} 7 & -178.5(2) & \mathrm{C} 12-\mathrm{C} 11-\mathrm{C} 16-\mathrm{Br} 2 & 177.20(17) \\ \mathrm{N} 1-\mathrm{C} 5-\mathrm{C} 6-\mathrm{C} 7 & -1.8(4) & \mathrm{N} 2-\mathrm{C} 11-\mathrm{C} 16-\mathrm{Br} 2 & -0.5(4) \\ \mathrm{C} 5-\mathrm{C} 6-\mathrm{C} 7-\mathrm{C} 1 & \mathrm{C} 4-\mathrm{C} 5-\mathrm{N} 1-\mathrm{N} 2 & -172.8(2) \\ \mathrm{O} 1-\mathrm{C} 1-\mathrm{C} 7-\mathrm{C} 6 & \mathrm{C} 6-\mathrm{C} 5-\mathrm{N} 1-\mathrm{N} 2 & 7.7(3) \\ \mathrm{C} 2-\mathrm{C} 1-\mathrm{C} 7-\mathrm{C} 6 & \mathrm{C} 5-\mathrm{N} 1-\mathrm{N} 2-\mathrm{C} 11 & 179.97(19) \\ \mathrm{C} 16-\mathrm{C} 11-\mathrm{C} 12-\mathrm{C} 13 & \mathrm{C} 12-\mathrm{C} 11-\mathrm{N} 2-\mathrm{N} 1 & 167.9(2) \\ \mathrm{N} 2-\mathrm{C} 11-\mathrm{C} 12-\mathrm{C} 13 & \mathrm{C} 16-\mathrm{C} 11-\mathrm{N} 2-\mathrm{N} 1 & -14.3(4) \\ \mathrm{C} 16-\mathrm{C} 11-\mathrm{C} 12-\mathrm{Br} 1 & 177.6(2) & & \\ \mathrm{N} 2-\mathrm{C} 11-\mathrm{C} 12-\mathrm{Br} 1 & 179.1(2) & & \\ -2.3(3) & 3.9(4) & & \end{array}$

Hydrogen-bond geometry $\left(A,{ }^{\circ}\right)$

\begin{tabular}{lllll}
\hline$D-\mathrm{H} \cdots A$ & $D-\mathrm{H}$ & $\mathrm{H} \cdots A$ & $D \cdots A$ & $D-\mathrm{H} \cdots A$ \\
\hline $\mathrm{C} 6-\mathrm{H} 6 \cdots \mathrm{N} 2$ & 0.95 & 2.36 & $2.705(3)$ & 101 \\
$\mathrm{O} 2-\mathrm{H} 2 \cdots \mathrm{O} 1$ & 0.84 & 2.1 & $2.591(2)$ & 117 \\
$\mathrm{C} 3-\mathrm{H} 3 \cdots \mathrm{O} 2{ }^{\mathrm{i}}$ & 0.95 & 2.54 & $3.241(3)$ & 131 \\
\hline
\end{tabular}

Symmetry code: (i) $-x+1, y-1 / 2,-z+3 / 2$. 